Estudios Románicos, Volumen 30, 2021, pp. 15-37

ISSN: 0210-4911

eISSN: 1989-614X

DOI: https://doi.org/10.6018/ER.470321

\title{
DE NUEVO SOBRE LA INTENSIFICACIÓN PRAGMÁTICA: REVISIÓN Y PROPUESTA*
}

(Revisiting pragmatic intensification: revision and definition proposal)

\author{
Marta Albelda Marco** \\ Maria Estellés Arguedas \\ Universitat de València-Val.Es.Co.
}

\begin{abstract}
This article proposes a characterisation of intensification from a pragmatic perspective, aiming to establish a comprehensive, delimited and unitary definition. This characterisation aspires to contribute to the recognition of the uses of intensification in real contexts and to help distinguish intensification from other similar phenomena. Before presenting the definition, the main contributions on the study of intensification are reviewed, both those conceived from a grammatical-semantic perspective and from a pragmatic perspective (the second are the most recent).

A cognitive and meta-representational perspective presents intensification as a linguistic attempt of a speaker to modify the assumptions that he/she attributes to the listener, and that do not meet this speaker's expectations. From a rhetorical perspective, the modification of the listener's assumptions pursues certain rhetorical effects, usually related to the achievement of local or global goals of the speaker in an ongoing interaction.
\end{abstract}

Keywords: Pragmatic intensification; Spanish; Rhetorical effects; Social effects; Cognition; Facework.

Resumen: Este artículo presenta una propuesta de caracterización de la intensificación desde una perspectiva pragmática con el objetivo de contribuir al establecimiento de una definición completa, delimitada y unitaria del fenómeno, que permita reconocer y discriminar los usos intensificaciones de otras categorías cercanas. Para ello, se revi-

* Esta investigación ha sido posible gracias a la ayuda recibida por el Ministerio de Ciencia e Innovación para el proyecto ESPRINT, "Estrategias pragmático-retóricas en la interacción conversacional conflictiva entre íntimos y conocidos: intensificación, atenuación y gestión interaccional" (ref. PID2020-114805GB-100).

** Dirección para correspondencia: Marta Albelda Marco / Maria Estellés Arguedas. Departament de Filologia Espanyola. Facultat de Filologia, Traducció i Comunicació. Universitat de València. Blasco Ibáñez 32.46010 Valencia (marta.albelda@uv.es; maria.estelles@uv.es). 
san las principales aportaciones previas al estudio del fenómeno: desde las más extendidas, de carácter gramatical y semántico, a las más recientes de naturaleza pragmática.

Se propone, por un lado, una explicación cognitiva y metarrepresentacional que define la intensificación como el intento lingüístico de un hablante por modificar los supuestos que le atribuye al oyente y que no responden a sus expectativas. Por otro lado, se considera que esta modificación de los supuestos del oyente persigue unos efectos retóricos determinados, normalmente relacionados con la consecución de metas locales o globales de los hablantes en una determinada interacción en curso.

Palabras clave: Intensificación pragmática; Español; Efectos retóricos; Efectos sociales; Cognición; Actividades de imagen.

\section{Introducción}

¿Qué es la intensificación en la lingüística? En las últimas décadas hemos asistido a un auge progresivo de estudios dedicados a este fenómeno, muchos de ellos enfocados desde diversas perspectivas lingüísticas. Son abundantes, por ejemplo, los trabajos sobre mecanismos gramaticales intensificadores, como morfemas derivativos, adverbios cuantificativos o intensivos, estructuras sintácticas de intensidad (consecutivas, cierto tipo de comparativas, etc.) o recursos fónicos (González Calvo 1984-1988, Ramos 1993, Dressler; Merlini 1994, Lavric 2106, Romero 2017, entre muchos otros). También se ha estudiado su valor semántico, como sema o clasema de intensidad, con efectos gradativos o cuantificadores (por ejemplo, Portero 1997, De Cesare 2002, Kennedy; Mc Nally 2005, Athanasiadou 2007, Margerie 2007). Se ha abordado desde el estudio de la modalidad epistémica y deóntica, como un modo de mostrar un mayor compromiso por parte de los hablantes con lo dicho o con lo sabido (Holmes 1982, Merlini 1986, Fuentes 1994, entre otros); también como una forma de reivindicar los derechos de primacía del conocimiento sobre los demás (Stivers 2005). Se ha empleado también el concepto en los estudios de cortesía y descortesía verbal, para explicar la manifestación lingüística de la cortesía valorizadora o el refuerzo de la carga amenazante de los mensajes, en el caso de la descortesía (Held 1989, Kerbrat-Orecchioni 1997, Maíz 2012, Uclés 2020).

Esta variedad de aplicaciones no hace más que poner en evidencia que es una noción transversal en la lingüística, puesto que a través de la lengua se pueden aumentar diversas propiedades en distintas dimensiones. La idea intuitiva de aumento, de refuerzo o superlación está en la base de los estudios mencionados, pero a partir de ahí, comienza la divergencia en las caracterizaciones.

Por su parte, el análisis y la visión pragmática de este fenómeno ha puesto de relieve su incidencia en la comunicación como estrategia intencional con fines retóricos. El presente trabajo aborda un estudio de la intensificación desde una perspectiva pragmática que contribuya a construir una base común al fenómeno en sus diversas aproximaciones y que facilite criterios para aunar posturas en la investigación a la hora de su reconocimiento.

Véanse, a continuación, cuatro muestras de habla real, todas ellas, en principio, con usos intensificadores pragmáticos, pero con efectos contextuales distintos en cada caso. 
En todos ellos, se marcan en negrita los fragmentos susceptibles de ser analizados como intensificación:

(1) Tres amigos conversan de forma familiar y distendida. En este momento se están refiriendo a un conocido cuya facilidad para aprobar el examen teórico para conducir les sorprende, ya que, a su juicio, no es una persona preparada para ello.

G: puees el chaval $\uparrow /$ o sea $\rightarrow$ / estaba estudiando EGB pero era pues/muy malo para estudiar ¿no? ceporro/ además que es un ANIMAL/ es muy BASTO yy $((\ldots))$

E: UN PELÓON/ ES UN PELÓON $\downarrow$ a la primera el carné $\downarrow$ la teoría y la práctica (...) pero si es corto $\downarrow$ cerrao $\downarrow$ mal criao $\downarrow$ yo qué sé

L: pues ¡vaya tela!

G: el chaval este/ o sea/ pues un día $\uparrow$ un día $\uparrow$ ¿no?/ decidió apuntarse a la autoescuela $\uparrow / y$ se apuntó a la autoescuela/ o sea creo que se salió en sexto/ no ha terminao nii-ni la EGB ino? ((...)) bueno él tenía en la cabeza que él iba a sacárselo/ fue a la autoescuela $\uparrow$ / su madre dice bueno/ si te vas a sacar el carnél por lo menos asiste a las clases§

E:

$\S$ claro

G: y él decía ¿ique yo asista a laas clases!? ((...)) yo no asisto a las clases ni nada $((\ldots))$ sin ir a la autoescuela para nada/ no ha ido para nada/ se leyó el libro $\uparrow$ en- en dos semanas $\uparrow((\ldots))$ es cantidad de cabezón $((\ldots))$

L: y aprobó el teórico

(Briz et al. 2002, Corpus Val.Es.Co. L.15.A. 2: 1308-1333) ${ }^{1}$

En el ejemplo (1) se reconocen marcas intensificadoras gramaticales en la descripción de las cualidades (negativas) de una tercera persona, orientadas a que se entienda lo sorprendente de que haya logrado su licencia de conducir en la primera convocatoria de las pruebas. Se recurre a la cuantificación superlativa de dichos rasgos con el adverbio muy, con adjetivaciones intensivas, enumeraciones, estructuras con el adverbio escalar $n i$, etc. En este contexto, la intención que mueve a los hablantes a intensificar parece ser argumentativa: se ofrecen pruebas que han de considerarse lo suficientemente fuertes para convencerse de lo asombroso del suceso.

(2) El periodista deportivo Tomás Roncero (R) comenta un reciente partido del Real Madrid en un programa televisivo de la cadena española La Sexta. Roncero está rodeado de seguidores de varios equipos de fútbol, entre ellos el presentador y un seguidor del Barça, quien ha afirmado previamente que el Real Madrid pasa por un momento difícil. R: Hoy el Madrid ha llegado diez, quince, veinte veces, ha podido meter, ha tenido a Hazard, que eso no falla nunca

(Tomás Roncero, El chiringuito de Jugones, La Sexta TVE, 22-10-2019).

En la tertulia deportiva de (2), $\mathrm{R}$ aporta varios argumentos en contra del supuesto de sus contertulios de que el Real Madrid está pasando por un momento crítico. Cada

1 Se proporciona al final del artículo una lista con las equivalencias de los signos de transcripción 
uno de los argumentos está intensificado internamente: se intensifica el número de veces que el equipo ha llegado a portería por medio de una escala ascendente (diez, quince, veinte veces) y se valora intensificadamente el papel de los jugadores (en particular de Hazard) con eso no falla nunca. De nuevo, se trata de una intensificación al servicio de la argumentación a favor del equipo del Real Madrid, con la que se rebate las posibles valoraciones de vulnerabilidad del equipo en la mente de los otros tertulianos. En este sentido, la intensificación se pone también al servicio de la reparación de la imagen del Real Madrid (y, como veremos, de R), que está en entredicho en esos momentos.

(3) Tres amigos, hombres y mujeres, conversan informal y distendidamente J: mira $\downarrow$ Láser Medicina/ hablando de láser

A: pos eso es lo que nesesito yo $\downarrow$ porque yo tengo el cuerpo to(do) etropeao $\downarrow$ si yo no voy mal encaminá (RISAS)

S: ¿tú tienes el cuerpo estropeado?

A: ¡OY QUE NO!

S: ¡tú flipas! (...) tú tienes un BUEN cuerpo/ ¡a lucirlo!

(Briz et al. 2002, Corpus Val.Es.Co., AP. 80: 853-857)

En la muestra (3), la hablante A, en su primera intervención, expresa una autocrítica (yo tengo el cuerpo to (do) etropeao $\downarrow$ si yo no voy mal encaminá), que además se reitera de forma intensificada en su segunda intervención (¡OY QUE NO!). En la última intervención, $\mathrm{S}$ ofrece su opinión contraria a la de A mediante una crítica intensificada: recurre a un verbo con valor de exageración (tú flipas), al énfasis prosódico en el adjetivo ponderativo (tú tienes un BUEN cuerpo), y al enunciado apelativo final (ja lucirlo!). Además de ser contraargumentos a la visión que de sí misma expresa A, se trata de un cumplido a su interlocutora y de una expresión de ánimo y buenos deseos. En suma, se aprecia un efecto contextual de cortesía valorizadora.

(4) Fragmento del programa de telerrealidad Gran Hermano 6, emitido en España por Telecinco. Los concursantes tienen una prueba y trabajan en equipos; E es la líder del grupo en el que está N. Están recibiendo encargos de comidas y tienen que prepararlas. En un determinado momento E se enfada y grita a $\mathrm{N}$ :

E: memoriza lo que yo te voy a decir/ ¿vale? Pascual/ confesionario $\uparrow /$ el nombre $\uparrow$ la dirección y el tipo de paella que quiere/ que es verduras/ ¿vale?// verduras $\uparrow$ al señor Pascual le gusta con pocos guisantes y más alcachofas

$\mathrm{N}$ : pero eso no hace falta que me lo digas a mí

E: ¿a quién se lo digo? ¿a quién se lo digo? / ¿VOY AL SÚPER Y SE LO DIGO AL SÚPER? ¿A QUIÉN SE LO DIGO?// YO ME ENCARGO DE HACERLO/ YO TE DIGO a ver el señor Pascual qué quierel ¿Y TÚ ME LO DICES?

$\mathrm{N}$ : ¿tú crees que yo te voy a aguantar asíl que me des voces o algo?/ pero tampoco necesito que me grites así $\rightarrow$

(Gran Hermano, Temporada 6, La Sexta TVE, 16/10/2005) 
En (4) los interlocutores hacen uso de mecanismos intensificadores, especialmente fónicos y sintácticos, mediante los cuales se transmiten efectos pragmáticos que en esta situación son precisamente contrarios a los del caso anterior, esto es, de hostilidad y descortesía verbal. E repite hasta tres veces su pregunta retórica retadora, con intensidad elevada. $\mathrm{N}$ reacciona con una pregunta retórica de curva melódica recriminante y acusadora hacia su interlocutora.

En los cuatro ejemplos anteriores se aprecia un común denominador en la expresión de una fuerza argumentativa reforzada y de un valor de intensidad elevada de lo dicho. Sin embargo, atendiendo a una perspectiva pragmática, los efectos situacionales y sus funciones retórico-argumentativas y socio-relacionales, como hemos visto, son divergentes.

¿Qué define, pues, a la intensificación pragmáticamente? Al abordar estudios particulares de análisis de casos no siempre se ha partido de una definición de la intensificación, puesto que se da por presupuesta en el terreno científico. No obstante, en los últimos años ya han visto la luz diversos trabajos que ofrecen propuestas de definición en el ámbito de la pragmática, como las de Holmes (1984), Meyer-Hermann (1988), Bazzanella et al. (1991), Saint-Pierre (1991), Briz (1997, 1998, 2017a, 2017b), Sbisà (2001), Albelda (2004, 2007, 2014), Bazzanella (2004), Margerie (2007), Gili Fivela; Bazzanella (eds., 2009), Schneider (2016), Fuentes (2015), Romero (2001, 2007, 2017), Bonacchi (2017), Fiorentini; Sansó (2017), Salzmann (2017), Beltrama (2018), Uclés (2020), Briz; Albelda (2021), entre otros. Muchas de ellas contienen rasgos comunes, pero al revisarlas y contrastarlas, se aprecia la diversidad y todavía vacilación en sus características, puesto que abarcan realidades lingüísticas no siempre coincidentes y no permiten reconocer los usos intensificadores con precisión en un contexto.

El presente artículo se propone ofrecer una propuesta de caracterización de la intensificación desde una perspectiva pragmática, y más en particular, centrada en una explicación desde la cognición y la retórica (apartado 3), donde se aíslan y exponen los rasgos básicos de su funcionamiento en cualquier uso lingüístico. Se reivindica su papel preponderantemente retórico y se plantea qué busca la intensificación cognitivamente. Previamente a ello se presenta una revisión del estado actual del fenómeno, atendiendo a las aportaciones que hasta ahora se han hecho desde la gramática, la semántica y la pragmática (apartado 2). La propuesta aquí ofrecida se apoya en los estudios anteriores y los integra, en especial respecto a los últimos avances realizados en el área de la pragmática. Por último, se aplica dicha propuesta a los ejemplos de partida para dar muestra de su viabilidad en usos intensificadores con diversos efectos contextuales (apartado 4).

\section{Estado actual del fenómeno de la intensificación. Caracterizaciones desde la gramática, la semántica y la pragmática}

\subsection{Aportaciones al estudio de la intensificación desde la gramática y la semántica}

La gramática tradicionalmente se ha referido a los fenómenos de intensidad bien mediante la gradación o mediante la cuantificación. Las propiedades, las cualidades, los entes, los eventos, los procesos, etc., se gradúan, y para lograrlo, la lengua dispone de 
numerosos mecanismos para expresarlo, entre ellos también formas y maneras novedosas, basadas en la creación léxica o morfológica. Son bien conocidos los procedimientos para graduar la cantidad y la cualidad de adjetivos, sustantivos, verbos o adverbios (Sapir 1944, Bolinger 1972, Alcina; Blecua 1975, Fernández Ramírez 1986, González Calvo 1984-1988, Herrero 1991, Ramos 1993, Dressler; Merlini 1994, Demonte, 1999, Sánchez López 1999, Romero 2001, 2017, Albelda 2007, entre muchos otros).

Tanto la gradación de la cualidad como la gradación de la cantidad (cuantificación) pueden hacerse en dos direcciones opuestas, descendente (atenuación, minimización) o ascendente (intensificación, maximización), y un mismo elemento puede recibir gradación de la cantidad (muchos años) y de la cualidad (súper año) (Bolinger 1972). Cuando la cuantificación o la gradación se expresan en su máximo punto se habla de superlación o ponderación (González Calvo 1984-1988).

No obstante, junto con la posibilidad de modificar intensivamente los elementos que afectan al contenido proposicional de la lengua, también está la de graduar los elementos modales. La intensificación mediante elementos modales tiene como objeto reforzar la aserción de lo dicho por los hablantes, bien sus valores de verdad o bien las opiniones o juicios de los hablantes. Se han estudiado también sobradamente en la literatura todos los recursos disponibles en las lenguas para la expresión de las escalas modales (Barrenechea 1969, Latour 1974, Holmes 1982, Merlini 1986, Martín Zorraquino 1992, Fuentes 1994, Briz 1997, Kovacci 1999, Romero 2017, entre otros), entre los cuales están algunas partículas discursivas y adverbios de la modalidad, en los que se aprecia la escala epistémica entre la incertidumbre y la seguridad (quizás, presuntamentel sin duda, evidentemente), y la escala de la modalidad deóntica, que expresa desde la posibilidad hasta la obligación (por suerte / forzosamente).

La intensidad es, pues, un valor semántico que cuenta con diversos modos gramaticales de expresión. En ese sentido, se entiende que se haya propuesto como un clasema más en el conjunto de componentes semánticos universales (Portero 1997). Sin embargo, como ya se ha estudiado, el fenómeno de intensificación parece ir más allá de la expresión de la gradación cuantificadora, de la superlación de la cualidad o de la valoración modal (Kleiber 2013).

Las descripciones gramaticales se ocupan de explicar la forma y el comportamiento de dicho valor semántico, pero no es su tarea entrar en la finalidad de uso de su empleo. Son los enfoques retóricos y pragmáticos los que podrán explicar las funciones que en la comunicación tienen dichos elementos semánticos.

Asimismo, cabe preguntarse si el hecho de que cualquier elemento que reciba una gradación de la cantidad o de la cualidad en forma ascendente superando un punto cero o de referencia es suficiente para considerarlo un caso de intensificación. En un número progresivo de estudios se aprecia que el término intensificación se está especializando en la caracterización de un fenómeno que desempeña funciones expresivas (y no solo informativas), de naturaleza subjetivo-evaluativa, y que tiene incidencia en la comunicación. En otras palabras, cada vez más, en la lingüística, intensificación alude al fenómeno pragmático.

Así pues, ¿podría decirse, por ejemplo, que cualquier uso del adverbio de cantidad muy o del adverbio intensivo auténtico, por el hecho de ser cuantificadores en una esca- 
la ascendente, provocaría automáticamente un efecto de intensificación? ¿Se considerarían intensificados los enunciados Tiene muy mal aspecto o Es un auténtico líder por el hecho de contener mecanismos de gradación semántica?

Atendiendo a una visión pragmática, la escalaridad semántica de por sí no sería rasgo suficiente para dar cuenta de los efectos retórico-pragmáticos, habría que ver estos elementos en un contexto y estudiar su función comunicativa (Albelda 2007, 2014). Tiene muy mal aspecto podría funcionar como una información descriptiva, sin otro valor pragmático, dada como respuesta a una persona que pregunta por teléfono "¿Cómo está hoy tu perrito?". Se trataría de una respuesta denotativa sin ninguna connotación añadida, a diferencia, quizás, de un enunciado como ;Qué mal aspecto tiene!, que sí contiene una evaluación apreciativa, unida a la gradación del adjetivo que supone la enunciación exclamativa y su partícula. Como ya apuntan las aproximaciones pragmáticas al fenómeno, a la escalaridad semántica se unen otros rasgos de subjetividad y evaluación (Albelda 2007, 2014, Athanasiadou 2007, Putska; Goldschmitt, eds. 2014, Nicola 2015, Lavric 2016, Mallogi 2017, Fiorentini; Sansó 2017, Beltrama 2018). Nos referimos a ello con más detalle en $\S 2.2$.

En resumen, como se aprecia a la luz de estas últimas cuestiones, no parece haberse alcanzado una definición suficientemente completa y específica para reconocer los usos intensificadores en sus respectivos contextos, y tampoco se ha logrado establecer los límites que la diferencian de otros fenómenos cercanos. Asimismo, siendo un mecanismo fundamental en la gestión interaccional, conviene seguir profundizando en su incidencia en la comunicación y en los valores pragmáticos que puede expresar. En el siguiente apartado, revisamos los avances pragmáticos logrados hasta ahora.

\subsection{Aportaciones al estudio de la intensificación desde la pragmática}

La investigación pragmática reciente ha ido apuntando el carácter estratégico de la intensificación al servicio de la negociación y de la eficacia comunicativa, y ha ampliado su ámbito de incidencia al enunciado y a la interacción. De hecho, el desarrollo del carácter pragmático de la intensificación (a menudo, en conjunción con el de la atenuación) viene de la mano de su aplicación a la gradación de la fuerza ilocutiva de los actos de habla y del refuerzo de los compromisos del emisor con lo dicho. La intensificación, frente a la atenuación, supone un aumento del grado de la fuerza ilocutiva (Holmes 1984, Labov 1984, Meyer Hermann 1988, Held 1989, Bazzanella et al. 1991, Sbisà 2001, Bazzanella 2004, Briz 1997, 1998, 2017, Albelda 2007, Kotwica 2015, Briz; Albelda 2021, etc.). Así pues, junto con la gradación proposicional, vemos que también se puede atenuar e intensificar en el nivel ilocutivo (la petición, la aserción, la queja, la disculpa, el agradecimiento, etc.).

Esta modificación de la fuerza ilocutiva de los enunciados tiene repercursiones a nivel retórico-pragmático en la comunicación. Se pone al servicio de la negociación conversacional y del logro de las metas interaccionales, para poder "asegurar la credibilidad, acuerdo o adhesión del destinatario" (Briz; Albelda 2021: 582).

Esta visión pragmática del fenómeno debe mucho a las caracterizaciones de la intensificación como un mecanismo de expresividad y subjetividad de la lengua. Ya Be- 
inhauer (1991 [1929]) se refirió a la intensificación como un fenómeno de realce de la figura del hablante y de su expresión afectiva, y Vigara Tauste $(1980,1992)$, como una modificación expresiva de los significados lingüísticos para lograr determinados efectos retóricos. Así, en una gran parte de la literatura, la intensificación se ha estudiado de manera conjunta con otros fenómenos retórico-expresivos como el énfasis, la mise en relief, el realce lingüístico, la subjetividad, la expresión de las emociones o la evaluación (véanse algunas revisiones sobre estas relaciones en De Cesare 2002, Albelda 2007, Kiesler 2014, Pustka 2014). De nuevo, la equiparación, o según los casos, intersección, de la intensificación con otro grupo de fenómenos, ahora ya no semánticos sino de naturaleza estilística y retórica, vuelve otra vez a mostrar la amplia versatilidad y alcance de su aplicación.

Se ha hecho necesario, por tanto, delimitar su papel y precisar los rasgos que caracterizan la intensificación a nivel comunicativo. Así, de acuerdo con Albelda $(2007,2014)$ y Briz; Albelda (2021), los efectos retóricos de la intensificación se han explicado como una implicatura bajo el concepto de evaluación o intención evaluativa. La intensificación pragmática supone la "intención evaluativa del emisor de expresar que se sobrepasan los supuestos esperados o establecidos como normales en una situación concreta, y que genera una implicatura en el contexto en el que se realiza" (Briz; Albelda 2021: 582). Se explica así que el punto de vista del hablante se haga presente en el enunciado y se exprese un juicio evaluativo de la realidad.

Se aprecia un interés y un progreso en el camino hacia la caracterización pragmática de la intensificación. Existe amplio consenso hacia su consideración como categoría pragmática de carácter estratégico en la comunicación, apuntada ya por Briz (1997, 1998) desde hace más de dos décadas. Asimismo, se desprende de los estudios previos su repercusión tanto en una dimensión retórico-argumentativa como en una dimensión social e interpersonal (Holmes 1984, Held 1989, Briz 1998, 2017a, 2017b, Brenes 2015, Fuentes 2016, Schneider 2017, Briz; Albelda 2021).

Como estrategia retórico-argumentativa se propone lograr la eficacia del habla y los objetivos comunicativos de los emisores. Está al servicio de la negociación interpersonal del acuerdo comunicativo y de la persuasión, y como hemos visto, busca la aceptación del mensaje por parte del interlocutor. Muy vinculada a la función retórica está su función social, pues la intensificación - al igual que la atenuación-contribuye al cuidado de las imágenes de los participantes en la comunicación y de las relaciones interpersonales entre los hablantes.

A continuación, recogemos una síntesis de lo que consideramos que son, hasta ahora, las principales aportaciones de los estudios previos a una caracterización pragmática de la intensificación. Estas servirán de base para la propuesta ofrecida en el apartado 3 y se incorporarán a ella. Así pues, apoyándonos en algunos trabajos clave en el estudio de la intensificación pragmática (entre otros, Holmes, 1984, Labov 1984, Meyer- Herman 1988, Held 1989, Bazzanella et al. 1991, Briz 1998, 2017a, 2017b, Sbisà 2001, Bazzanella 2004, Albelda 2007, 2014, Margerie 2007, Schneider 2017, Fuentes 2016, Beltrama 2018, Briz; Albelda 2021), se puede decir que se apuntan como rasgos fundamentales de su caracterización los siguientes: 
(i) Se forma sobre un valor semántico escalar superándolo gradualmente; esto es, parte de una escala semántica en la que se señala el grado máximo posible de una dimensión, pero lo supera porque (ii)

(ii) Se añade a (i) una intención evaluativa por parte del hablante de sobrepasar los supuestos esperados en una situación concreta.

(iii) Se expresa a través de elementos proposicionales y modales. Como se ha visto en el apartado § 2.1, estos se asocian a la graduación de la cantidad o de la cualidad semántica o a la expresión de formas de modalidad epistémica o deóntica con las que el hablante se compromete con lo dicho en el segmento proposicional o aumenta las obligaciones contraídas hacia su interlocutor.

(iv) Es (también) una estrategia pragmática; esto es, no solamente afecta a lo dicho, sino a lo implicado, y tiene consecuencias sobre el mero acto de enunciación y los participantes; así, no solo aumenta la prominencia del acto mismo de comunicación — más allá del contenido del mensaje—, sino que también da claves sobre las emociones y actitudes de los participantes y las relaciones entre ellos.

(v) Sirve a funciones de doble naturaleza: retórico-argumentativas y sociales; esto es, por un lado, lleva al logro o la consecución de metas y, por otro, gestiona las relaciones interpersonales entre los participantes en el acto comunicativo.

(vi) Como consecuencia de lo anterior, se considera un fenómeno que debe reconocerse contextualmente; es una implicatura, bien convencional bien conversacional, puesto que cada lengua desarrolla mecanismos lingüísticos que permiten transmitir esta intención pragmática, y con el tiempo, algunos de ellos logran convencionalizarse.

En las líneas que siguen presentaremos brevemente una propuesta de definición operativa de intensificación, que se apoya en los aspectos pragmáticos previos. Esta propuesta se centra en una explicación cognitiva del fenómeno y en su predominante incidencia retórica. Apuntamos criterios para su reconocimiento y, tras ello, volveremos sobre los ejemplos iniciales para reinterpretarlos en estos nuevos términos.

\section{Propuesta de caracterización desde una perspectiva cognitiva y retórica}

El ensamblaje y revisión bajo un mismo marco de los aspectos caracterizadores de la intensificación pragmática en los que se ha avanzado hasta ahora (§ 2.2), da cuenta de cómo funciona en el terreno lingüístico y comunicativo, explica cómo se canaliza en la lengua y ofrece varias pautas sobre su repercusión en la comunicación. Siendo estas valiosas contribuciones al estudio del fenómeno, dejan pendientes algunos puntos que podrían completar su explicación, facilitarían un reconocimiento más transparente y

2 Así, se considera que la escalaridad semántica no es rasgo suficiente para explicar los efectos retóricopragmáticos que generan estos fenómenos. Si bien su base semántica es escalar, es necesario contar con otros rasgos que explican las implicaturas pragmáticas que generan en la comunicación. 
directo en su uso contextual, y permitirían deslindar la intensificación de otros fenómenos con los que intersecta.

Diferentes propuestas han establecido diferentes límites, de modo que, como se ha visto más arriba, se han considerado casos de intensificación, entre otros, algunos fenómenos de cuantificación semántica (Tiene muy mal aspecto). Los límites se pueden situar en diversos umbrales y, sin duda, crear con ellos una visión teórica coherente, pero la presente propuesta opta por establecer la línea divisoria entre la intensificación y otros fenómenos cercanos de cuantificación y expresividad en su carácter metacognitivo o metarrepresentacional, en el sentido de que implica un cálculo previo de los supuestos del interlocutor y trata de modificar esos supuestos.

La perspectiva cognitiva, y más en concreto, metarrepresentacional, ofrece un ángulo más de la descripción del fenómeno, lo que ayuda a delimitarla todavía mejor y a establecer más criterios y más concretos para su reconocimiento. Desde esta perspectiva vamos a explicar cómo opera la mente del hablante cuando recurre a la intensificación en el uso conversacional. Como se verá, la operación cognitiva de la intensificación tiene su base en una necesidad retórica de la comunicación.

La intensificación, en estos términos, podría definirse como el intento (para)lingüístico de un hablante por modificar un supuesto que le atribuye al oyente y que no responde a sus expectativas. Ello implica, por tanto, dos fases: una, en la que el hablante calcula qué piensa su interlocutor sobre un aspecto X y otra, en la que trata de actuar (para)lingüísticamente para modificarlo. Esta modificación de los supuestos del oyente persigue unos efectos retóricos determinados, normalmente relacionados con la consecución de metas locales o globales de la interacción en curso. Se manifiesta típicamente a través de expresiones marcadas que aumentan la cantidad, calidad o prominencia de alguno de los aspectos del enunciado y del acto de la enunciación. Los aspectos fundamentales de esta definición, de carácter metarrepresentacional y retórico, como hemos visto, se desarrollarán brevemente a continuación, con un énfasis especial en el primero, por ser, quizás, el menos explorado en la bibliografía. ${ }^{3}$

La idea de metarrepresentación está presente, de manera más o menos directa, en el estudio de muchos fenómenos de intensificación, aunque no necesariamente bajo esta etiqueta. Sperber y Wilson (1986: 220-221), por ejemplo, apuntan que un fenómeno como la repetición en ejemplos como He went for a long, long walk; There's a fox, a fox in the garden o My childhood days are gone, gone, tiene funciones muy diversas, pero todas ellas tienen en común que, al generar un sobrecoste de procesamiento (pues hay una parte que se repite), deben resultar en un aumento de los efectos cognitivos, y así lo interpreta el oyente. En este caso, el sobrecoste de la repetición hace al oyente ver que el fragmento marcado es "more relevant than the hearer would have spontaneously realised" (Sperber y Wilson 1986: 21) o que una cualidad, estado, etc., se da en una medida mayor "than the hearer would otherwise have thought" (Sperber y Wilson 1986: 220).

$3 \quad$ El tercer aspecto, las formas concretas de expresión lingüística de la intensificación, se dejarán al margen en este trabajo por dos razones: su multiplicidad, que haría el artículo innnecesariamente extenso, y el hecho de que se trata del aspecto más trabajado previamente, puesto que los trabajos centrados en formas o conjuntos de formas son los más frecuentes en la bibliografía especializada. 
Se observa, por tanto, cómo la utilización de un recurso 'antieconómico' como repetir es interpretado por el oyente como una llamada a replantearse la cantidad o intensidad de la que sería su interpretación neutra, si no estuviera esa repetición.

Asimismo, visto desde la perspectiva especular, la del hablante, cabe preguntarse cuál es la finalidad de utilizar este mecanismo. Consideramos que el hablante parte de que es necesario un cálculo previo de lo que piensa (o pensaría) su interlocutor antes de ofrecerle un enunciado con una forma lingüística marcada, sea mediante una repetición, un aumento de la prominencia fónica, la gradación máxima de una magnitud o estado dentro de una escala, o en suma, cualquier recurso lingüístico de lo que estamos considerando intensificación. Así, veamos la diferencia entre (5) y (6), en los que se encuentra el mismo mecanismo formal, la presencia de un 'muy'. El caso de (5) es un fragmento extraído de un vídeo de un youtuber español en el que habla sobre la pigmentación de una paleta de acuarelas

(5) (...) con una ligera tonalidad pues vamos a decir avioletada es que no sé es que no sé si la vais a apreciar en la / en la cámara luego los verdes también me han encantado/ he de decir que me encantan/ luego están ahí los brillantes que tienen muy buena pinta/ así parece que pigmentan bastante bien a la primera (My yellow journal, ACUARELAS SCHMINCKE/ Unboxing \& Lámina..., 25/10/2020. https://www.youtube.com/watch?v=MUO8C41SWWc 12:54)

Por su parte, el fragmento (6), está extraído del talent show Maestros de la Costura, y recoge el momento de la valoración de una prenda por parte de un miembro del jurado. La prueba debía desarrollarse por parejas y la jueza felicita a uno de los participantes, G, por haber elegido a su compañero, A, que es a quien se debe el mérito. A ha realizado la parte del conjunto que está bien (la falda), mientras que $G$ ha realizado la parte de arriba, que está mal confeccionada. En un momento dado, $G$ dice a la jueza, M, que irá mejorando poco a poco, que de momento lo ha felicitado por elegir compañero, pero luego todo se andará.

(6) $\mathrm{M}: \mathrm{G} /$ tengo que felicitarte

G: sorpréndeme

M: felicidades por haber escogido a A

G: ah pues gracias muchas [gracias]

M: [has sidô] súperlisto/ nada tiene que ver la confección de la falda/ con la confección del cuerpo/// o sea la falda está IMPECABLE y el cuerpo $\rightarrow$ tiene mucho que desear

G: sí

M: el escote no está bieen $\uparrow$ la sisa está regular no está pulidaa/ las pinzas regulao sea está/ MAL confeccionada§

$\mathrm{G}$ :

felicitado por elegir=]

M:

$\S$ bueno poquito a poco/ [de momento me has

[o sea considero $\uparrow$ sí] 
$\mathrm{G}:=\mathrm{a} A \uparrow /$ todo se andará

M: eso es /MUY/ importante/ en la vida real/ rodearse de un equipo $(\ldots)$

(Maestros de la costura, TVE. Temporada 4, episodio 2 (38:33))

La diferencia entre (5) y (6) estriba en la presencia o ausencia, respectivamente, de ese cálculo metarrepresentacional: en (5), la hablante simplemente define los comentarios como "muy buena pinta" para señalar que son esos los que le gustan. Sin embargo, en (6), la situación es más compleja (por eso se ha presentado un contexto tan extendido): con el comentario de G, "bueno poquito a poco/ de momento me has felicitado por elegir a $\mathrm{A} \uparrow /$ todo se andará", la jueza del programa, $\mathrm{M}$, entiende que $\mathrm{G}$ considera el hecho de saber elegir un compañero un mérito menor. Esta asunción de $\mathrm{G}$ no está de acuerdo con las expectativas de M, que replica diciendo "eso es /MUY/ importante/ en la vida real/ rodearse de un equipo", precisamente para modificar el supuesto que atribuye a G a partir de sus palabras, que elegir buen compañero de equipo no es un mérito menor, sino algo muy importante en el día a día de la industria de la moda. Por supuesto, este "MUY" aparece, además, en conjunción con una fuerte prominencia fónica manifestada a través de una delimitación de "muy" entre pausas y de un aumento de su intensidad.

El carácter metarrepresentacional o el cálculo y gestión de los supuestos atribuidos al oyente es un rasgo que la intensificación comparte con otros fenómenos interaccionales como la atenuación $\left.{ }^{4}\right)$. Sin embargo, hay dos diferencias importantes:

a) En la atenuación, tras el cálculo de los supuestos del interlocutor, el hablante está conforme con los pensamientos que le atribuye. Esto es, el hablante cree que la opinión de su interlocutor se ajusta a sus expectativas, de modo que el recurso a la atenuación lo que se propone es precisamente evitar que esa opinión 'positiva' que le atribuye pueda cambiar a raíz de una amenaza potencial (por ejemplo, una acción que podría comprometer su imagen, como admitir que le gusta curiosear cuando pasa al lado de un accidente de tráfico: "Solo estaba interesándome un poquitín por lo que ha pasado"). Sin embargo, la intensificación se produce cuando el cálculo previo lleva al hablante a pensar que los supuestos del oyente no son los que él espera; por tanto, debe modificarlos para que se ajusten a sus expectativas. La atenuación es, pues, una estrategia conservadora o preservadora, mientras que la intensificación es innovadora o modificadora.

b) En la atenuación, no todos los tipos de supuestos del oyente que el hablante calcula previamente $-\mathrm{o}$ que el hablante metarrepresenta— son importantes; únicamente tienen importancia los supuestos del oyente que afectan a la imagen del hablante: el hablante trata de calcular únicamente qué piensa el oyente de él/

$4 \quad$ Sobre este tema, se podrá consultar este trabajo de las autoras actualmente en preparación: "Mitigation revisited. An operative and integrated definition of the pragmatic concept, its strategic values and its linguistic expression". 
ella y en qué manera sus acciones futuras pueden alterar ese (buen) concepto ${ }^{5}$. En la intensificación, en cambio, se calculan los supuestos del oyente acerca de un aspecto dado, esté o no relacionado con la autoimagen del hablante.

Así pues, la frontera entre la intensificación semántica -por tanto, más bien, gradación(como en (5)) y pragmática (como en (6)) viene marcada por la presencia del cálculo metarrepresentacional de supuestos. Dentro de la intensificación pragmática, los supuestos atribuidos al oyente que el hablante desea modificar pueden afectar a su autoimagen o no hacerlo; así, hablaremos de intensificación con y sin imagen, de las cuales solo la primera, con imagen, constituiría un reflejo especular de la atenuación. Nótese, además, que la intensificación con imagen, en paralelo con lo que sucede en la atenuación, siempre implica la presencia de autoimagen (que, además en algunos casos, también incluirá el cuidado de la imagen de los otros): el hablante modifica aquellos supuestos que reflejan una imagen suya no deseada. Y, también como en la atenuación, en ocasiones hace uso estratégico de la cortesía para lograr sus metas; esto es, en ocasiones es necesario acudir al cuidado de la imagen del interlocutor para garantizar o mejorar la imagen propia (Figura 1) .

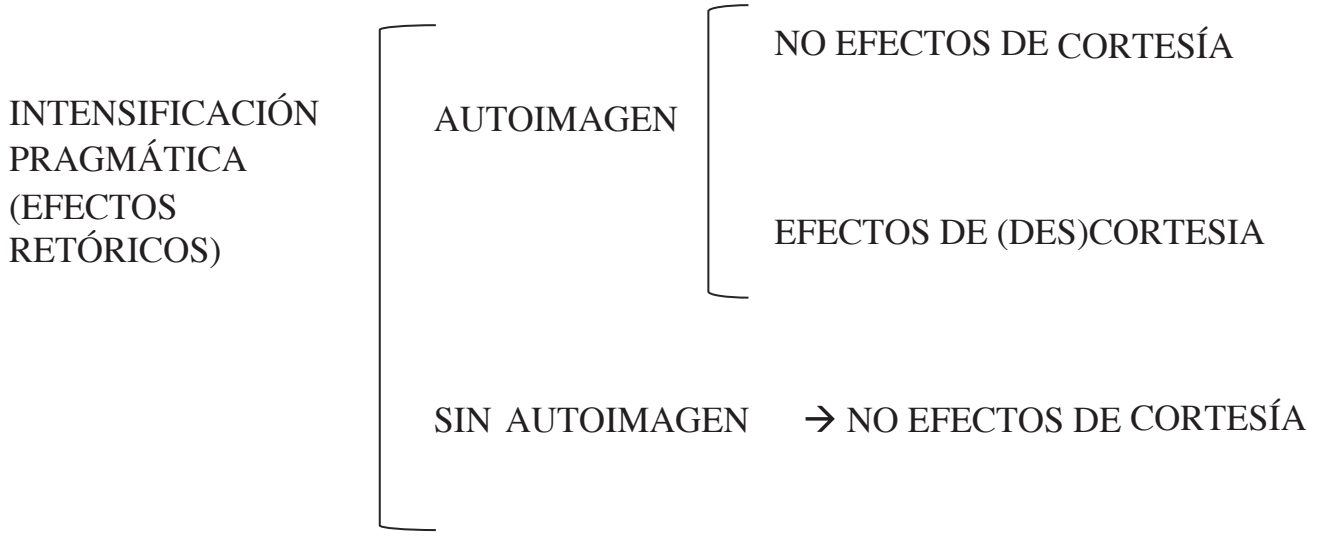

Figura 1. Tipos de intensificación

La figura refleja, tal y como se explica a continuación, que los efectos retóricos surgen siempre del uso de la intensificación, mientras que los sociales (cortesía e imagen), no siempre son efectos contextuales de la intensificación. Asimismo, como veremos con más detalle en el apartado 4, los cuatro ejemplos iniciales ilustran cada una de estas posibilidades de intensificación. En el ejemplo (1) donde se describe la conducta de una tercera persona no presente en la conversación, consideramos que la intensificación no afecta ni a la imagen del hablante (autoimagen) ni a la de los interlocutores (y, por ello, no tiene efectos de cortesía); es un caso de intensificación sin autoimagen. El ejemplo (2) sí implica la imagen del

\footnotetext{
$5 \quad$ Véanse, en este sentido, Kerbrat-Orecchioni (1991, 2004, 2005).

6 Para una ampliación de este punto, véase el trabajo citado en nota 4.
} 
periodista, Roncero; hay por tanto, autoimagen, pero no se relacionan con objetivos (des) corteses. Por último, en los ejemplos (3) y (4) la intensificación surge por protección de la autoimagen: en (3), además, sirve a efectos corteses y en (4), a fines descorteses.

La segunda de las ideas planteadas en la presente propuesta está, en gran medida, presente en la bibliografía y podría decirse que hoy ya es indisputable; se trata de la visión retórica de la intensificación. Se plantea la intensificación como un mecanismo que persigue un logro local o global en la interacción: los supuestos del oyente se quieren modificar para algo. En la línea de Briz; Albelda (2021), la intensificación sirve a funciones retórico-argumentativas y sociales, relacionadas con la consecución de metas (por ejemplo, convencer a alguien para que haga algo) o con la gestión de las relaciones entre los participantes en la comunicación hasta lograr que se encuentren en el punto deseado por el hablante (lograr que se el hablante sienta halagado y por tanto la relación se estreche, etc.). Los fines son, por tanto, múltiples, pero la idea común subyacente a todos es que el hablante entiende que, para poder llegar felizmente a la consecución de su meta, es necesario que su oyente tenga (o adquiera) unos determinados supuestos y, cuando calcula que su oyente no tiene esos supuestos -o que los tiene, pero sin la fuerza suficiente-, el hablante actúa para modificarlos, ajustarlos a sus expectativas y crear, por tanto, el terreno propicio para que dichas metas puedan lograrse.

En relación con lo anterior, se ha dicho que siendo la intensificación un fenómeno comunicativo al servicio tanto de fines retóricos como sociales, al igual que la atenuación, sin embargo, la intensificación presenta predominantemente una incidencia retórico-argumentativa, al centrarse prioritariamente en la figura del yo-hablante. Por su parte, la atenuación, posibilitando también ambos fines, se centra preponderantemente en los fines sociales, pues se focaliza la figura del tú-interlocutor (Briz; Albelda 2021). Se entiende así, también, que haya usos intensificadores que sean sin imagen o que no sirvan a efectos (des)corteses, pues no siempre surge por fines sociales, mientras que sí surge siempre por fines retóricos.

Las consecuencias de esta modificación de los supuestos en el interlocutor suelen ser a mejor en el nivel argumentativo, pues el hablante se hace -o busca hacerse- más fuerte argumentativamente. Sin embargo, en el nivel social las consecuencias pueden ser absolutamente opuestas: bien pueden estrechar las relaciones (como se ve en el ejemplo 3 , cortesía valorizadora), o bien reforzar las heridas, distanciar los vínculos interpersonales (como se aprecia en el ejemplo 4, con efectos descorteses). En ese sentido, se ha de considerar que la intensificación es un arma de doble filo en la comunicación relacional. Así, incorporada a actos de habla agradadores y de cortesía verbal, puede favorecer el fortalecimiento de los vínculos sociales (Briz 1998, 2017, 2018, Albelda 2003, 2007, Maíz-Arévalo 2012, Bello 2015, Romero 2017: 197-220, Barros 2018a, 2018b), mientras que, incorporada a los actos de habla hostiles, la intensificación incrementa la agresividad comunicativa, por lo que sus efectos interpersonales son nocivos (Fuentes y Alcaide 2008, Brenes 2015, Uclés 2020), aunque no por ello no pretendidos por el hablante (que puede querer lograr que el interlocutor se enfade, por ejemplo).

Junto con estas dos coordenadas explicativas, la de carácter cognitivo y la de carácter retórico, hay que contar con la expresión lingüística (y paralingüística, que no estudiaremos en este trabajo) de la intensificación, que, como se ha visto en $\S 2.1$., es, sin duda, la parte 
más desarrollada del estudio de la intensificación pragmática, puesto que la mayoría de los trabajos dedicados a ella son semasiológicos y parten del estudio de formas concretas.

Así, el inventario de formas y estructuras a las que se les atribuye un carácter intensificador es extenso, y está constituido fundamentalmente por mecanismos que aumentan la cantidad, calidad o prominencia de un fragmento y, sobre todo, la prominencia del acto mismo de enunciación: dado que el hablante quiere cambiar los supuestos que atribuye a su oyente, es el primer interesado en que su mensaje y su voluntad de emitirlo queden meridianamente claros y sean perfectamente perceptibles para su oyente. Como consecuencia, se utilizan formas de expresión marcada que suelen estar relacionados con la superlación, la repetición, la expresión del punto máximo de una escala, etc., al tiempo que se emplean mecanismos que ponen de relieve la prominencia del mensaje, como una prosodia prominente (pitch alto, ritmo más lento, intensidad más alta, pronunciación enfática o marcada, etc.).

La manera de reconocer los usos intensificados en un contexto partirá normalmente de la identificación de las formas lingüísticas, a las cuales seguirá la identificación del cálculo cognitivo de los hablantes y el análisis de las metas retóricas interaccionales. El cálculo de los supuestos del oyente es una operación casi inmediata y natural para los hablantes en el momento de la comunicación, pero es compleja en el análisis post-hoc, y por tanto, cabe ajustar la metodología para delimitar los ejemplos que tienen intensificación pragmática (según la propuesta aquí planteada) de los que no. Por eso, es fundamental encontrar los criterios (i) y (ii) y, en la medida de lo posible, también (iii):

i. la presencia de marcas lingüísticas (o paralingüísticas, cuando se disponga de vídeo) de incremento en la cuantificación o en la cualidad, la superlación, la prominencia, etc.,

ii. la existencia explícita de un cotexto que justifique claramente que el hablante ha calculado un desajuste entre sus expectativas y los supuestos del oyente, y que le hace obrar en consecuencia por medio de la emisión de (i)

iii. la persecución de una meta global o local. Para su análisis se precisa disponer de un contexto suficientemente amplio que permita acceder a los fines interaccionales que los hablantes se proponen en su interacción. Muy frecuentemente este punto no es rastreable en los ejemplos fragmentarios que aquí hemos ofrecido, especialmente para la identificación de las metas globales como la mejora de las relaciones sociales, preparación del terreno para garantizarse una buena opinión del oyente, etc.

En el siguiente apartado se retoman los ejemplos de partida para aplicar la propuesta de caracterización cognitivo-retórica de la intensificación.

\section{Aplicación de la propuesta. Análisis de los ejemplos iniciales}

Veamos la aplicación de la propuesta anterior a los ejemplos de la introducción. En todos ellos se aprecia que la explicación cognitiva básica es coincidente, mientras que difieren en sus efectos retóricos y sociales. 
En el ejemplo (1), los hablantes $\mathrm{G}$ y E (ahora, $\mathrm{H}$ ) emplean diversos usos intensificados dirigidos a modificar los supuestos que su amiga $\mathrm{L}$ (ahora, $\mathrm{O}$, oyente) tiene respecto al "chaval" (X, una tercera persona ausente en la conversación) del que están hablando. G y E han calculado que $\mathrm{L}$ no se asombrará lo suficiente al conocer que $\mathrm{X}$ ha aprobado a la primera el carné de conducir. Con el objetivo retórico de convencerla de que no es algo esperable sino sorprendente, pretenden modificar su visión sobre las capacidades de $\mathrm{X}$ mediante formas marcadas de intensificación. En el siguiente esquema se detalla el punto de partida en la mente de los hablantes (i) y el punto final (ii) tras el recurso a la intensificación (iii):

i) $\mathrm{H}$ cree que $\mathrm{O}$ piensa $\mathrm{Y}$

$[\mathrm{Y}=\mathrm{L}$ no es consciente de las exiguas capacidades personales que $\mathrm{X}$ tiene para aprobar el examen de coche]. NO ACORDE CON LAS EXPECTATIVAS DE $\underline{\mathrm{H}}$

ii) $\mathrm{H}$ despliega estrategias lingüísticas intensificadoras para que los supuestos que atribuye a sus $\mathrm{O}$ cambien:

es muy malo (...) ceporrol además que es un ANIMAL/ es muy BASTO (...) UN PELÓONI ES UN PELÓON (...) es corto $\downarrow$ cerrao $\downarrow$ mal criao $\downarrow$ yo qué sé (...) no ha terminao ni la EGB (...) sin ir a la autoescuela para nadal (...) es cantidad de cabezón

iii) $\mathrm{H}$ cree que $\mathrm{O}$ piensa $\mathrm{Y}^{\prime}$

$\left[\mathrm{Y}^{\prime}=\right.$ 'X es una persona muy poco capaz y sin dotes para aprobar el examen de coche, por lo que haberlo superado en el primer intento es una gran contraexpectativa']. ACORDE CON LAS EXPECTATIVAS DE H

En el ejemplo (2), Roncero (hablante, $\mathrm{H}$ ) ha calculado que la situación de partida de sus oyentes $(\mathrm{O})$ sobre la valoración del Real Madrid, no es deseable o, dicho de otro modo, no se ajusta a sus expectativas. Los mecanismos intensificadores que emplea se dirigen a cambiar esos supuestos de partida de sus interlocutores, con los que no está de acuerdo. Las fases de su cálculo cognitivo y su intervención en la comunicación para modificar el pensamiento de sus contertulios serían las siguientes:

i) $\mathrm{H}$ cree que $\mathrm{O}$ piensa $\mathrm{Y}$ [Y= 'el Real Madrid está en una mala situación deportiva']. NO ACORDE CON LAS EXPECTATIVAS DE $\mathrm{H}$

ii) $\mathrm{H}$ despliega estrategias lingüísticas intensificadoras para que los supuestos que atribuye a sus $\mathrm{O}$ cambien:

Hoy el Madrid ha ganado diez, quince, veinte veces, ha podido meter, ha tenido a Hazard, que eso no falla nunca

iii) $\mathrm{H}$ cree que $\mathrm{O}$ piensa $\mathrm{Y}^{\prime}$

[Y' = 'el Real Madrid está en una buena/no tan mala situación deportiva']. ACORDE CON LAS EXPECTATIVAS DE H

Esta modificación de los supuestos mentales del oyente persigue un claro fin retórico, contraargumentar la posición de sus contertulios sobre este equipo de fútbol. 
También persigue un fin social de protección de la autoimagen del hablante, Roncero, que se ha visto amenazada, al ser él un claro hincha del Real Madrid.

Como hemos visto en la Figura (1) y en la presentación de los ejemplos en la introducción, en (1) y (2), a diferencia de (3) y (4), el uso de la intensificación, además de producir efectos retóricos en la interacción, surte también efectos sociales de cortesía valorizadora en (3) y de descortesía y hostilidad en (4).

En el ejemplo (3) se señalaron usos intensificados tanto en la hablante A como en el hablante S. Nos centraremos ahora en el enunciado "tú flipas (...) tú tienes un buen cuerpo/ ¡a lucirlo!" de la última intervención de S, reconocida en su contexto como un halago (face flattering act) hacia A. Esta, en su primera intervención, se autocritica delante de sus amigos ("yo tengo el cuerpo to(do) etropeao $\downarrow$ si yo no voy mal encaminá"). Le sigue una negociación sobre la valoración de su cuerpo, que concluye con la última intervención de $\mathrm{S}$ en la que intensifica su opinión contraria a la de $\mathrm{A}$. De esta forma, $\mathrm{S}$ pretende resarcir y compensar la lesión pública de autoestima que se ha autoatribuido su interlocutora. El recorrido cognitivo de $\mathrm{S}$ (ahora $\mathrm{H}$, hablante), con su metarrepresentación de las creencias de A (ahora $\mathrm{O}$, oyente) es el siguiente:

(i) $\mathrm{H}$ cree que $\mathrm{O}$ piensa $\mathrm{Y}$

$[\mathrm{Y}=$ 'Mi interlocutor admite que tengo el cuerpo estropeado, por lo que es un mal amigo'] NO ACORDE CON LAS EXPECTATIVAS DE $\mathrm{H}$

ii) $\mathrm{H}$ despliega estrategias lingüísticas intensificadoras para que los supuestos que atribuye a sus $\mathrm{O}$ cambien:

tú flipas (...) tú tienes un buen cuerpol ja lucirlo!

iii) $\mathrm{H}$ cree que $\mathrm{O}$ piensa $\mathrm{Y}^{\prime}$

$\left[Y^{\prime}=\right.$ ' $\mathrm{Mi}$ interlocutor no admite que me autoatribuya una cualidad negativa, porque es falsa, por lo tanto, es un buen amigo']. ACORDE CON LAS EXPECTATIVAS DE H

Por último, en el ejemplo (4), a diferencia del (3), la intensificación no busca un efecto social y relacional positivo entre los interlocutores, sino que provoca un ataque reforzado de la imagen del interlocutor, a quien se desacredita. El ataque se concreta en que Nicky imputa a Eloísa una omisión de deberes -en doble sentido- a través de una recriminación intensificada: deberes de conocimiento, se le acusa de no saber cómo debe tratar a su interlocutor, y deberes de incongruencia en la acción, porque debería haber actuado conforme a ese conocimiento y no haberle hablado así.

Veamos cómo Nicky (ahora $\mathrm{H}$, hablante) en su última intervención recurre a la intensificación con el propósito de modificar los supuestos cognitivos que atribuye a Eloísa (ahora O, oyente) sobre sí mismo.

i) $\mathrm{H}$ cree que $\mathrm{O}$ piensa $\mathrm{Y}$ [Y = 'acepto/tolero que me hablen con gritos y me traten con menosprecio'] $\underline{\mathrm{NO}}$ ACORDE CON LAS EXPECTATIVAS DE H

ii) $\mathrm{H}$ despliega estrategias lingüísticas intensificadoras para que los supuestos que atribuye a sus $\mathrm{O}$ cambien:

¿Tú crees que yo te voy a aguantar así, que me des voces? 
iii) $\mathrm{H}$ cree que $\mathrm{O}$ piensa $\mathrm{Y}^{\prime}$

[Y'= 'no acepto ni tolero que me hablen con gritos y me traten con menosprecio']. ACORDE CON LAS EXPECTATIVAS DE H

Los efectos de la intensificación en este fragmento de Gran Hermano son sociales (al igual que en la muestra 3), pero, como se ha explicado en el apartado anterior ( $\$$ 3 ), son prioritariamente retórico-argumentativos. De la pregunta " ¿Tú crees que yo te voy a aguantar así que me des voces o algo?" se implica una afirmación reforzada del contenido proposicional ('no te voy a aguantar así); Nicky no va a admitir gritos ni una conducta despótica hacia él. Se intensifica, en este caso, la obviedad, se implica de forma intensificada que la interlocutora debería conocer a priori la información que se le recrimina. Este efecto retórico, a su vez, logra el efecto social de ataque y desacreditación de la imagen del interlocutor.

El análisis de estos ejemplos a la luz de la propuesta de intensificación presentada muestra que lo común en todos ellos es la explicación cognitiva. En los cuatro ejemplos se reconoce que el hablante calcula un desajuste entre sus expectativas y los supuestos de su oyente, y para ello recurre a estrategias de intensificación que le permitirán intervenir en la modificación de los supuestos de su interlocutor. Esta definición de naturaleza cognitiva, junto con la definición retórica de contribuir al logro de las metales locales o globales de los hablantes en una interacción, constituyen dos aspectos comunes, a nuestro juicio, en todo uso intensificador. Así, a las características y explicaciones pragmáticas ofrecidas hasta ahora para este fenómeno, se unen ahora los rasgos aquí propuestos. Ha sido, pues, nuestro objetivo contribuir a una mayor perfilación de sus rasgos, de forma que se pueda ir acotando cada vez más la definición y el reconocimiento unificado de la intensificación.

\section{BIBLIOGRAFÍA}

ALBELDA, Marta (2007): La Intensificación como categoría pragmática: revisión y propuesta. Berna: Peter Lang.

(2014): Escalaridad y evaluación: rasgos caracterizadores de la intensificación pragmática, Putska, Elissa/Goldschmitt, Stefanie (eds.), Emotionen, Expressivität, Emphase. Berlín: Erich Schmidt Verlag, 79-94.

ALBELDA MARCO, Marta; BRIZ GÓMEZ, Antonio (2021): "Atenuación e intensificación”, Escandell Vidal, María Victoria et al. (eds.), Pragmática. Akal: Madrid, 567-590.

ALCINA FRANCH, Juan; BLECUA PERDICES, José Manuel (1975): Gramática española. Barcelona: Ariel.

ATHANASIADOU, Angeliki (2007): "On the subjectivity of intensifiers". Language Sciences 29: 554-565.

BARRENECHEA, Ana María (1979 [1969]): “Operadores pragmáticos de actitud oracional: los adverbios en -mente y otros signos", Ana María Barrenechea et al. (eds.): Estudios lingüísticos y dialectológicos. Buenos Aires: Hachette Universidad, 39-59. 
BARROS GARCÍA, María Jesús (2018a): Cortesía valorizadora. Uso en la conversación informal española. Frankfurt am Main: Peter Lang.

(2018b): "Face-enhancing compliments in informal conversations in Valencian Spanish". Borealis. An International Journal of Hispanic Linguistics 7(1): 147-168.

BAZZANELLA, Carla (2004): "Atténuation et intensification en Italien: dimensions et configuration pragmatique", Maria Helena Araújo Carreira (ed.), Plus ou moins?! L'atténuation et l'intensification dans les langues romanes. Saint-Denis: Université Paris 8 Vincennes Saint-Denis, 177-203.

BAZZANELLA, Carla; CAFFI, Claudia; SBISÀ, Marina (1991): "Scalar dimensions of illocutionary force", Igor Ž. Žagar (ed.). Speech acts: fiction or reality? Proceedings of the International Conference, Ljubljana, Yugoslavia, November 15, 1990. Ljubljana: Inštitut za družbene vede, 63-76.

BEINHAUER, Werner (1991 [1929]): El español coloquial. Madrid: Gredos.

BELLO MESERVE, Bethany (2015): Atenuación e intensificación pragmáticas en la expresión de actividades de imagen: un estudio contrastivo en conversaciones coloquiales del español y del inglés. Tesis Doctoral, Valencia: Universidad de Valencia.

BELTRAMA, Andrea (2018): "Totally Between Subjectivity and Discourse. Exploring the Pragmatic Side of Intensification". Journal of Semantics 35(2): 219-261.

BOLINGER, Dwhigt (1972): Degree Words. La Haya: Mouton.

BONACCHI, Silvia (2017): "What does reduplication intensify? The semantics and pragmatics of reduplicated forms in Italian and their equivalents in German", Maria Napoli; Miriam Ravetto (eds.), Exploring Intensification: Synchronic, diachronic and cross-linguistic perspectives. Studies in Language Companion Series 189. Amsterdam: John Benjamins, 289-303.

BRENES, Ester (2015): "La intensificación de la aserción en el parlamento andaluz. Análisis pragmalingüístico de los verbos de opinión". Cultura, Lenguaje y Representación 14: 9-31.

BRIZ GÓMEZ, Antonio (1997): "Los intensificadores en la conversación coloquial”, Antonio Briz Gómez, José Ramón Gómez Molina, María José Martínez Alcalde, Grupo Val. Es. Co (eds.), Pragmática y gramática del español hablado. Actas del II Simposio sobre análisis del discurso oral. Valencia: Universidad de Valencia, 13-36.

(1998): El español coloquial en la conversación. Esbozo de pragmagramática. Barcelona: Ariel.

(2017a): "Una propuesta funcional para el análisis de la estrategia pragmática intensificadora en la conversación coloquial", Marta Albelda; Wiltrud Mihatsch (eds.), Atenuación e intensificación en géneros discursivos. Madrid: Iberoamericana Vervuert, 43-67.

(2017b): "Otra vez sobre las funciones de la intensificación en la conversación coloquial". Boletín de Filología, 52 (2): 37-58. 
DE CESARE, Anna-Maria (2002): Intensification, modalisation et focalization. Les different effects des adverbs proprio, davero et veramente. Berna: Peter Lang.

DEMONTE, Violeta (1999): "El adjetivo: clases y usos. La posición del adjetivo en el sintagma nominal", Ignacio Bosque; Violeta Demonte (coords.), Gramática descriptiva de la lengua española. I. Madrid: Espasa 129-215.

DRESSLER, Wolfgang U.; Lavinia MERLINI BARBARESI (1994): Morphopragmatics. Diminutives and intensifiers in Italian, German, and other languages. Berlin: Mouton De Gruyter.

FERNÁNDEZ RAMÍREZ, Salvador (1986): Gramática española. 3.1. El nombre (volumen preparado por J. Polo). Madrid: Arco / Libros.

FIORENTINI, Ilaria; SANSÓ, Andrea (2017): "Intensifiers between grammar and pragmatics. A lesson from a language contact situation", Maria Napoli; Miriam Ravetto (eds.), Exploring Intensification: Synchronic, diachronic and cross-linguistic perspectives. Studies in Language Companion Series 189. Amsterdam: John Benjamins, 173-192.

FUENTES RODRÍGUEZ, Catalina (1994): "Usos discursivos y orientación argumentativa: de hecho, en efecto, efectivamente", Español Actual 64: 5-18.

(2015): "Pragmatic grammar of es que: The intensification marker". Estudios Filológicos 55(55):53-76.

(2016): "Atenuación e intensificación estratégicas", Catalina Fuentes, Estrategias argumentativas y discurso político, Madrid: Arco/Libros, 163-22.

FUENTES, Catalina; ALCAIDE, Esperanza (2008): (Des) cortesía, agresividad y violencia verbal en la sociedad actual. Sevilla: Universidad Internacional Andaluza.

GILI FIVELA, B; BAZZANELLA, C. (eds.). (2009): Fenomeni di intensità nell'italiano parlato. Firenze: Franco Cesati.

GONZÁLEZ CALVO, José Manuel (1984-1988): "Sobre la expresión de 'lo superlativo' en español I, II, III, IV y V”, Anuario de Estudios Filológicos VII, 1984, pp. 172-205; VIII, 1985, pp. 113-146; IX, 1986, pp. 129-143; X, 1987, pp. 101132 y XI, 1988, pp. 159-174.

HELD, Gudrun (1989): "On the role of maximization in verbal politeness". Multilingua 8 (2-3): 167-206.

HERRERO MORENO, Gemma (1991): "Procedimientos de intensificación-ponderación en el español coloquial”. Español Actual 56: 39-51

HOLMES, Janet (1982): "Expressing doubt and certainty in English". RELC Journal 13 (2): 9-28.

(1984): "Modifying illocutionary force". Journal of Pragmatics 8: 345-365.

KENNEDY, Chris; McNALLY, Louise (2005): "Scale structure, degree modification and the semantics of gradable predicates". Language 81(2): 345-381.

KERBRAT-ORECCHIONI, Catherine (1991): "La politesse dans les interactions verbales", en Stati, S., Weigand, E., Hundsnurscher, F. (eds.): Dialoganalyse III, vol. 1, Tübingen: Niemeyer, pp. 39-59. 
(1997): "A multilevel approach in the study of talk-in-interaction". Pragmatics, 7, pp. 1-18.

(2004): “¿Es universal la cortesía?”, en Bravo, D., Briz, A. (eds.): Pragmática sociocultural: estudios sobre el discurso de cortesía en español, Barcelona: Ariel, pp. 39-53.

(2005): Le discours en interaction, Paris: A. Colin.

KIESLER, Reinhard (2014): "Expressivité et emphase", Pustka, Elissa; Stefanie Goldschmitt (eds.), Emotionen, Expressivität, Emphase. Berlin: Erich Schmidt Verlag, 41-56.

KLEIBER, Georges (2013): “À la recherche de l'intensité”. Langue Française, 177 (marzo 2013), pp. 63-76.

KOTWICA, Dorota (2015): “Al parecer evidencial atenuante ¿Y reforzador?”, Álvarez, Garrido, González (eds.), Jóvenes aportaciones a la investigación lingüística. Sevilla: Alfar, 481-494.

KOVACCI, Ofelia (1999): "El adverbio", Ignacio Bosque; Violeta Demonte (coords.), Gramática descriptiva de la lengua española. I. Madrid: Espasa, 705-786.

LABOV, William (1984): "Intensity", en D. Schiffrin (ed.): Meaning, form and use in context: Linguistic applications, Washington: Georgetown University Press, pp. 43-70.

LAVRIC, Eva (2016): “Ay señor/ qué juventud esta”, Wiltrud Mihastch y Marta Albelda (eds.): La atenuación y la intensificación desde una perspectiva semánticopragmática. Revista Internacional de Lingüística Iberoamericana XVI: 33-54.

LATOUR, Bernd (1974): Elative und affirmative modälworter. Hamburgo: Buske.

MAÍZ ARÉVALO, Carmen (2012): "Was that a compliment? Implicit compliments in English and Spanish". Journal of Pragmatics 44 (8), 980-996.

MARGERI, Hélène (2007): "From Downgrading to (over)intensifying: A Pragmatic Study in English and French", Ivan Kecskes; Laurence Horn (eds.), Explorations in Pragmatics. De Gruyter Mouton, 287-312.

MARTÍN ZORRAQUINO, María Antonia (1992): "Partículas y modalidad", Günter Holtus et al. (eds.): Lexikon der Romanistichen Linguistik VI, 1. Tubinga: Niemeyer, 110-124.

MERLINI BARBARESI, Lavinia (1986): “'Obviously' and 'certainly': two different functions in argumentative discourse", Ferenc Kiefer (ed.), Folia Linguistica. Acta Societatis Linguisticae Europeae. La Haya: Mouton, 3-24.

MEYER-HERMANN, Reinhard (1988): "Atenuación e intensificación (análisis pragmático de sus formas y funciones en español hablado)". Anuario de Estudios Filológicos XI: 275-290.

GRANDI, Nicola (2015): "The place of evaluation within morphology", Nicola Grandi; Livia Kortvelyessy (eds), The Edinburgh Handbook of Evaluative Morphology. Edinburgh: EUP, 74-90.

PORTERO, Carmen (1997): Intensificación: estudio de un clasema. Tesis doctoral. Córdoba: Universidad de Córdoba. 
PUSTKA, Elissa (2014): “Was ist Expressivität?”, Pustka, Elissa; Stefanie Goldschmitt (eds.), Emotionen, Expressivität, Emphase. Berlin: Erich Schmidt Verlag, 11-39.

PUSTKA, E.; GOLDSCHMITT (eds.) (2014): Emotionen, Expressivität, Emphase. Berlin: Erich Schmidt Verlag.

RAMOS MÁRQUEZ, María del Mar (1993): "La intensificación del adjetivo y del adverbio en el discurso (sintaxis oral)", Catalina Fuentes Rodríguez (ed.), Sociolingüistica andaluza 8. Sevilla: Universidad de Sevilla, 183-213.

ROMERO, Clara (2001): L'intensité en français contemporain: analyse sémantique et pragmatique. Tesis doctoral. Paris: Université de Paris 8. (2007): "Pour une définition générale de l'intensité dans le langage". Travaux de Linguistique: Revue Internationale de Linguistique Française 54: 57-68. (2017): L'intensité et son expression en français. Paris: Ophrys.

SAINT-PIERRE, Madeleine (1991): "Illocutoire et modalisation: les marqueurs d'intensité en français". Revue québécoise de linguistique 20: 223-236.

SALZMANN, Katharina (2017): " A pragmatic view on intensification: Expansions in German and Italian“, Maria Napoli; Miriam Ravetto (eds.), Exploring Intensification: Synchronic, diachronic and cross-linguistic perspectives. Studies in Language Companion Series 189. Amsterdam: John Benjamins, 231-250.

SÁNCHEZ LÓPEZ, Cristina (1999): "Los cuantificadores: clases de cuantificadores y estructuras cuantificativas". Ignacio Bosque; Violeta Demonte (coords.), Gramática descriptiva de la lengua española. I. Madrid: Espasa, 1025-1128.

SAPIR, Edward (1944): "Grading, a study in Semantics". Philosophy of Science 2: 93-116. SBISÁ, Marina (2001): "Illocutionary force and degrees of strength in language use". Journal of Pragmatics 33: 1791-1814

SCHNEIDER, Stefan (2017): "Las dimensiones de la intensificación y de la atenuación”, Marta Albelda; Wiltrud Mihatsch (eds.), Atenuación e intensificación en diferentes géneros discursivos. Madrid: Iberoamericana/Vervuert, 23-42.

SPERBER, Dan; Deirdre WILSON (1994 [1986]): La relevancia. Comunicación y procesos cognitivos. Madrid: Visor.

STIVERS, Tania (2005): "Modified Repeats: One Method for Asserting Primary Rights from Second Position". Research on Language and Social Interaction 38(2):131-58.

UCLÉS RAMADA, Gloria (2020): "Mitigation and Boosting as Face-Protection Functions". Journal of Pragmatics 169: 206-18.

VIGARA TAUSTE, Ana María (1980): Aspectos del español hablado. Madrid: SGEL. (1992): Morfosintaxis del español coloquial (Esbozo estilístico). Madrid: Gredos.

\section{PERFIL ACADÉMICO Y PROFESIONAL}

Marta Albelda es catedrática de Lengua Española en la Universitat de València y miembro del grupo Val.Es.Co. Se doctoró en 2004 con una tesis sobre intensificación pragmática y ha desarrollado su investigación en diversas facetas de la pragmática y 
semántica del español hablado, en sociolingüística y lingüística de corpus. Ha participado en múltiples proyectos sobre pragmática y sociolingüística del español y ha dirigido cuatro de ellos sobre atenuación e intensificación, los dos últimos junto con María Estellés (Es.Vag.Atenuación y ESPRINT). Es, asimismo, coordinadora del corpus Ameresco de conversaciones coloquiales.

Maria Estellés es doctora en Lengua Española por la Universitat de València (2009) y actualmente es profesora titular en el departamento de Filología Española de dicha institución. Miembro del grupo de investigación Val.Es.Co., sus intereses se centran en la pragmática del español hablado, atenuación, evidencialidad, corpus lingüísticos y marcadores del discurso. Junto a Marta Albelda dirige el proyecto Es.VaG.Atenuación, sobre la atenuación lingüística en su variación genérica, y con ella coordina, asimismo, el corpus Ameresco de conversaciones coloquiales de las principales ciudades de América Latina y España. Asimismo, codirige también con Marta Albelda el proyecto ESPRINT sobre la interacción conflictiva y su relación con la intensificación y la gestión interaccional.

Fecha de recepción: 23/03/2021

Fecha de aceptación: 31/05/2021

\section{ANEXO}

\section{Sistema de transcripción}

Los signos fundamentales del sistema de transcripción del corpus de Val.Es.Co. que se emplean en el presente artículo, son los siguientes:

$\S \quad$ Sucesión inmediata, sin pausa apreciable, entre emisiones de distintos hablantes.

$=\quad$ Mantenimiento del turno de un participante en un solapamiento.

[ Lugar donde se inicia un solapamiento o superposición.

] Final del habla simultánea.

- $\quad$ Reinicios y autointerrupciones sin pausa.

I Pausa corta, inferior al medio segundo.

// Pausa entre medio segundo y un segundo.

III Pausa de un segundo o más.

(5") Silencio (lapso o intervalo) de 5 segundos; se indica el $n^{\circ}$ de segundos en las pausas de más de un segundo, cuando sea especialmente significativo.

$\uparrow \quad$ Entonación ascendente.

$\downarrow \quad$ Entonación descendente.

$\rightarrow \quad$ Entonación mantenida o suspendida.

PESADO Pronunciación marcada o enfática (dos o más letras mayúsculas).

(()) Fragmento indescifrable.

((...)) Interrupciones de la grabación o de la transcripción. 